\title{
Fusion with exotic nuclei using a microscopic approach
}

\author{
K. Vo-Phuoc ${ }^{1, \star}$, C. Simenel ${ }^{1}$, and E. C. Simpson ${ }^{1,}$ \\ ${ }^{1}$ Department of Nuclear Physics, Australian National University, ACT 2601, Australia
}

\begin{abstract}
Fusion reactions near the barrier energy are studied in this proceedings with the Hartree-Fock (HF) method, on systems involving ${ }^{40-54} \mathrm{Ca}+{ }^{116,132} \mathrm{Sn}$. Both static and time-dependent (TDHF) versions of the method are used to probe what structure effects play a role in the fusion barrier energy. In static HF calculations for fusion, ground state properties of the nuclei influence the barrier energy. When dynamics are added in with TDHF, some effects from static properties (for example, the neutron skin) disappear. To understand the role of vibrations in dynamic reactions, TDHF is used in conjunction with the coupled-channels approach. Discussion on the effects of transfer is also presented.
\end{abstract}

\section{Introduction}

Properties of nuclides near the neutron dripline are often not well known. Moreover, how neutron-rich nuclides interact in reactions remains an open question as it is often unfeasible to study this experimentally. How the nuclear structure of these nuclei affects heavy-ion fusion reactions is of strong interest, with upcoming exotic beam facilities and SHE formation studies.

Effects of internal nuclear structure on heavy-ion fusion can be seen by studying features of experimental fusion barrier distributions [1, 2]. Experiments have shown that dynamic effects such as low-lying vibrational couplings [3-5] and transfer reactions [6-10] strongly affect fusion reactions. Other dynamic effects, such as breakup [11-14] and rotational couplings [4], have also been seen to play a role.

The conventional choice of method for studying heavy-ion fusion was the coupled-channels (CC) approach, which needs input parameters of the structure of the nuclei in the reaction. When these parameters are not known or experimental data is unavailable, especially for very neutron rich nuclei, a microscopic approach is more appealing.

The Hartree-Fock (HF) method is a self-consistent theory which is a useful tool to study both nuclear structure and reactions. By contrast to $\mathrm{CC}$ methods, the only input is the nucleon-nucleon interaction. The time-dependent version (TDHF) allows for the study of dynamical effects on fusion reactions. TDHF has been used for several purposes for nuclear structure and reactions (see Refs. [1517] for reviews). Modern codes solve the TDHF equation in three dimensions and include spin-orbit interaction [1821]. Recent applications include vibration [22-26], fusion [27-34], transfer [35-39], quasifission [40-46] and fission [47-52] studies.

\footnotetext{
^e-mail: kirsten.vo-phuoc@anu.edu.au
}

The TDHF method for studying reactions includes many dynamical effects simultaneously. This makes it difficult to isolate the effect of a single dynamical coupling on a fusion reaction. The method of including microscopic inputs to coupled-channels (CC) analysis [28, 53] is a way to isolate certain dynamic effects, and is used to unravel the TDHF dynamics and the influence of these on the fusion reactions in this proceedings.

\section{Numerical details}

The Hartree-Fock (HF) method assumes nucleons in a nucleus are independent particles that generate a self consistent mean-field. The only input into the HF method is the nucleon-nucleon interaction, often taken to be the Skyrme interaction. This is a major advantage of the method as no prior knowledge of the structure or dynamical modes of nuclei are needed for calculations. In this proceedings, two Skyrme parametrisations were used, the SLy4d [18] and UNEDF1 [54] parametrisations. These two in particular were chosen as they have no correction for centre of mass motion and are therefore suitable for dynamic calculations involving two different nuclei $[16,55]$.

The TDHF equation reads

$$
[h[\rho], \rho]=i \hbar \frac{d \rho}{d t},
$$

where $\rho$ is the one-body density matrix of the independent particle system and $h[\rho]$ is the HF hamiltonian. Before any dynamical calculations using equation (1) can be made for reactions or vibrations, individual nuclei in their $\mathrm{HF}$ ground state must first be calculated using the static version of equation (1) where the right hand side is equal to zero. The Ev8 code [56] is used for calculating HF ground states. The box size we used for a HF ground state nucleus is $(2 \times 11.2)^{3} \mathrm{fm}^{3}$ assuming three planes of symmetry. 
Pairing in the HF ground state is accounted for at the BCS level with a density-dependent delta pairing force $[57,58]$ in the form of

$$
\hat{v}_{\text {pair }}\left(\tilde{\mathbf{r}}_{1}, \tilde{\mathbf{r}}_{2}\right)=\tilde{t_{0}} \delta\left(\tilde{\mathbf{r}}_{1}-\tilde{\mathbf{r}}_{2}\right)\left(1-\frac{\rho(\tilde{\mathbf{R}})}{\tilde{\rho}_{0}}\right),
$$

with parameter values $t_{0}=1000 \mathrm{MeV} . \mathrm{fm}^{3}$ and $\tilde{\rho}_{0}=0.16$ $\mathrm{fm}^{-3}$.

As for reactions calculations (both static and dynamic), the box size is increased to $67.2 \times 22.4 \times(2 \times 11.2) \mathrm{fm}^{3}$ in the $x-y-z$ orientation with the $x$ axis being the collision axis. Only central collisions are studied in this work. There is now only one plane of symmetry, $z=0$. The maximum starting distance between two nuclei was $44.8 \mathrm{fm}$.

In dynamic reaction calculations, the TDHF equation (1) is solved by the TDHF3D code [18]. A mean field is now defined for the entire system, generated by all the nucleons. The single-particle occupation numbers induced by pairing correlations are kept constant in the dynamics. A Galilean boost, $e^{i \mathbf{k}_{j} \cdot \hat{\mathbf{r}}}$, giving each nucleon in nucleus $j$ momentum, is applied to the system at the initial time which moves the nuclei towards each other.

In dynamic vibration calculations, the box size is for a single nucleus, with dimensions $22.4 \times 22.4 \times(2 \times 11.2) \mathrm{fm}^{3}$. Only one plane of symmetry is present again, at $z=0$. The TDHF3D code is also used to evolve the nucleus with response to a multipole boost at initial time, $e^{-i \varepsilon \hat{Q}_{\lambda}}$ where

$$
\hat{Q}_{\lambda}=\sum_{i=1}^{A} r^{\lambda} Y_{\lambda 0}(\hat{r})
$$

and with boost intensity $\varepsilon$ small enough to be in the linear regime. More details on the vibrational boost is given in Section 4.2.

\section{Static effects}

For static HF reactions, the density distributions of the two nuclei, $\rho_{1}$ and $\rho_{2}$, are frozen in their HF ground state at all points of the reaction. The frozen HF potential $[59,60]$ between them, $V(\mathbf{r})$, is calculated. For the nuclear part of the potential, the sum of the energies is used,

$$
V_{N}(\mathbf{r})=E(\mathbf{r})-E_{\mathrm{HF}}\left[\rho_{1}\right]-E_{\mathrm{HF}}\left[\rho_{2}\right],
$$

where the energy of the total system is

$$
E(\mathbf{r})=\int \mathcal{E}\left[\rho_{1}\left(\mathbf{r}^{\prime}\right)+\rho_{2}\left(\mathbf{r}-\mathbf{r}^{\prime}\right)\right] d \mathbf{r}^{\prime}
$$

The HF energy for each nucleus is

$$
E_{\mathrm{HF}}\left[\rho_{j}\right]=\int \mathcal{E}\left[\rho_{j}(\mathbf{r})\right] d \mathbf{r},
$$

where $\mathcal{E}$ is the Skyrme energy density. This approach does not account for Pauli repulsion which can be important inside the barrier [53]. Here, we neglect this effect on the barrier energy, $V_{B}$.

In Fig. 1, the static barriers, $V_{B}$, for ${ }^{40-54} \mathrm{Ca}+{ }^{116} \mathrm{Sn}$ are shown by the dashed lines for the SLy4d (red, closed

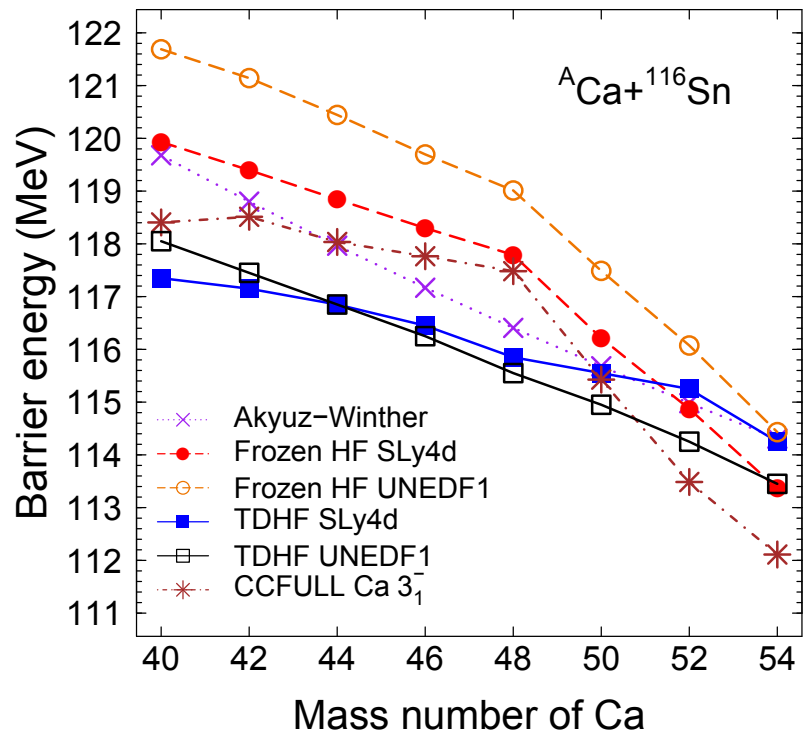

Figure 1. Barrier energies for reactions ${ }^{40-54} \mathrm{Ca}+{ }^{116} \mathrm{Sn}$ comparing static HF, TDHF, AW and CCFull (with the $3_{1}^{-}$state of calcium) calculations. For the HF calculations, both SLy4d and UNEDF1 parametrisations are shown.

circles) and UNEDF1 (orange, open circles) parametrisation alongside the dotted line of Akyüz-Winther (AW) potential [61] barriers (purple crosses) for comparison. The most noticeable difference between the static HF barriers and the AW barriers is that the latter shows a near constant decrease of the fusion barrier energy as neutron number is increased and the static HF calculations show a change of gradient in the barrier energies before and after the ${ }^{48} \mathrm{Ca}$ projectile. Also note that the UNEDF1 parametrisation yields a higher barrier than the SLy4d parametrisation, by $2 \mathrm{MeV}$ for a ${ }^{40} \mathrm{Ca}$ projectile and by $1 \mathrm{MeV}$ for a ${ }^{54} \mathrm{Ca}$ projectile.

To explain what causes this changing gradient in both the static $\mathrm{HF}$ lines for $V_{B}$, we consider the root-meansquare (rms) radius in the calcium projectiles. This is a static property arising from the HF ground state. Both neutron and proton rms radii for the calcium chain are shown in Fig. 2 (solid and dashed lines respectively) for both parametrisations. It can be seen that in the neutron rms radius (solid line), there is a change of gradient before and after ${ }^{48} \mathrm{Ca}$. An increase in nuclear radius induces a decrease of the barrier energies. This is because the point at which the fusion barrier occurs between the nuclei will be at a larger distance thereby decreasing the Coulomb potential and hence the overall fusion barrier. The single particle shells of the neutrons helps to explain why the neutron radius increases more rapidly after ${ }^{48} \mathrm{Ca}$. $\mathrm{As}{ }^{48} \mathrm{Ca}$ is a doubly magic nucleus the energy gap between these shells is substantial. The neutrons in the $2 p$ shells filled after $N=28$ are less bound, meaning their radial wavefuncion extends to a larger radius than the neutrons in the $1 f_{\frac{7}{2}}$ shell $(N \leq 28)$ which gives rise to a more rapid increase of neutron rms radius. 


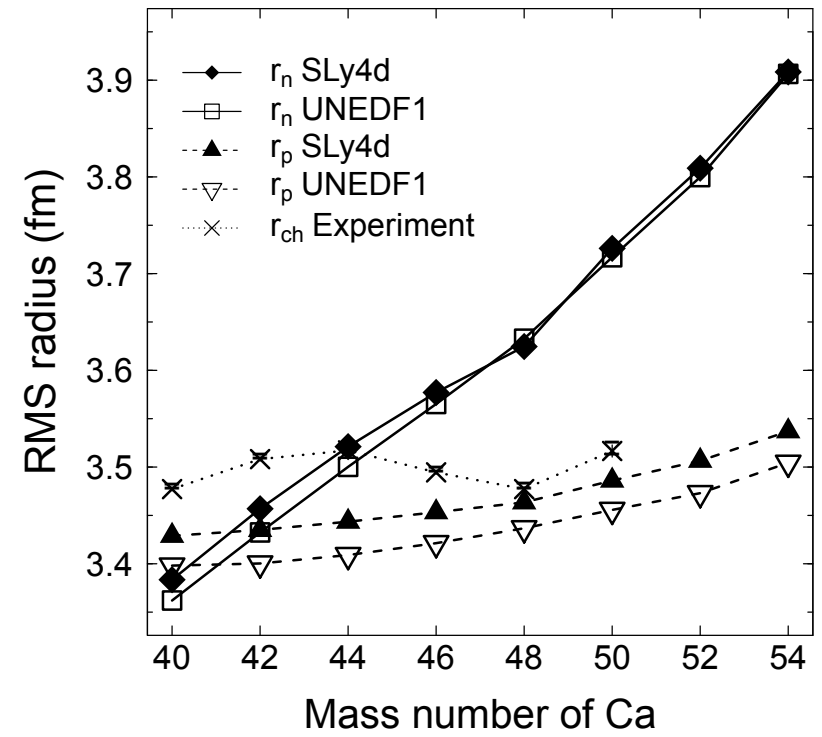

Figure 2. Calcium isotope proton (dashed line), neutron (solid line) rms radii in the SLy4d and UNEDF1 parametrisations. Also shown are the experimental charge rms radii.

Also included in Fig. 2 are the experimental charge rms radii (dotted line, crosses) [62, 63]. These values are relatively close to the calculated proton radii though the deviations are larger for mid-shell nuclei due to correlations not accounted for at the mean-field level [64]. If HF reproduced these values for the proton radii of these calcium isotopes then the expected fusion barrier for these systems would be at slightly lower energies.

\section{Dynamic effects}

\subsection{Barriers}

Freezing nuclei in their ground state is not a realistic way to simulate fusion reactions. Dynamical couplings must be included to allow nuclei to be excited from their ground state, so for calculating reactions the TDHF equation (1) is used, outlined in Section 2.

In TDHF, as there is only a single, average trajectory for a reaction, the fusion probability is either 0 or 1 . For reactions involving calcium on tin, if a compound nucleus has formed and remained compact for $5 \mathrm{zs}$, it is classified as having successfully fused. The TDHF fusion barrier or "threshold" is found by incrementally increasing the energy for a system until fusion is observed [60].

The TDHF fusion thresholds are shown by the solid lines in Fig. 1, for SLy4d (blue, closed squares) and UNEDF1 (black, open squares). We see that including dynamics, in most cases, reduces the static fusion barrier. The exception is for ${ }^{52,54} \mathrm{Ca}+{ }^{116} \mathrm{Sn}$ in the SLy4d parametrisation, where the dynamic barrier is actually higher than the static barrier. Whilst the dynamic barrier for UNEDF1 is not higher than its static counterpart for the two heaviest systems, the difference between the two HF barriers as neutron number increases reduces consistently. Over-

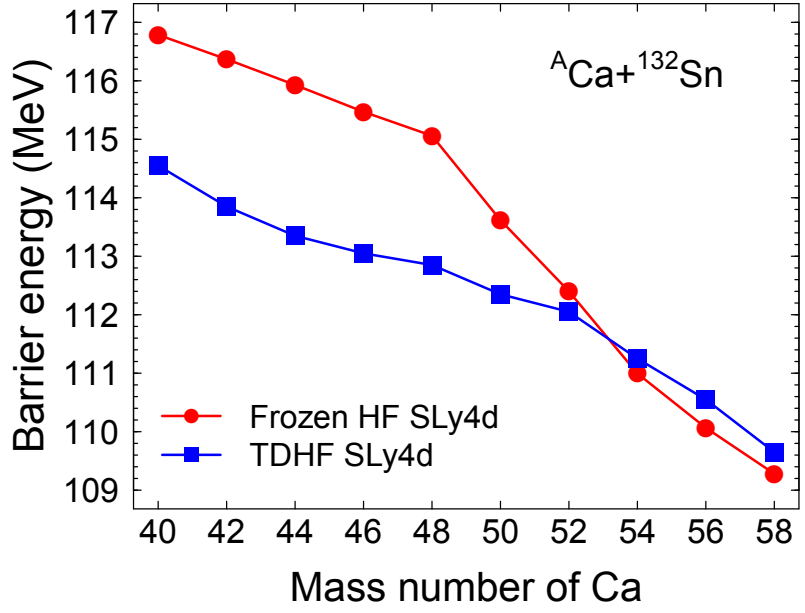

Figure 3. Static and dynamic HF barrier energies with ${ }^{132} \mathrm{Sn}$ as the heavy fragment, using the SLy4d parametrisation.

all, the barrier is reduced more for UNEDF1 parametrisation. This could indicate that the dynamical modes in this parametrisation are stronger than for the SLy4d parametrisation. We also observe that including dynamics washes out the static structure effects as the TDHF barriers no longer have a change of gradient after the ${ }^{48} \mathrm{Ca}$ nucleus.

The expected dynamical effects present in these TDHF calculations are vibrations and transfer. As all the nuclei ground states were spherical or near-spherical, we do not expect coupling to rotational states to play a major role in these reactions. The goal now is to unravel the dynamics in the TDHF calculations to see which dynamical effects have an impact on fusion thresholds.

Although the main focus of the work is for ${ }^{40-54} \mathrm{Ca}+{ }^{116} \mathrm{Sn}$, we performed similar calculations for the systems ${ }^{40-58} \mathrm{Ca}+{ }^{132} \mathrm{Sn}$ shown in Figure 3. There is also a crossover present between static and dynamic barriers from ${ }^{54} \mathrm{Ca}+{ }^{132} \mathrm{Sn}$ onwards.

\subsection{Vibration effects}

It is well known that low-lying octupole phonons play an important role in fusion, in particular in reactions with ${ }^{40} \mathrm{Ca}$ [65]. Bearing this in mind, coupling to the $3{ }_{1}^{-}$state of the calcium isotopes was considered for these reactions within the CC approach. Quadrupole vibrations could also impact fusion, however, the low-lying $2^{+}$states in ${ }^{40} \mathrm{Ca}$ are not collective. The study of the couplings to $2_{1}^{+}$states in the other calcium isotopes will be the subject of future work.

An octupole boost taking $\lambda=3$ for the operator in equation (3) is applied to the HF ground state of the calcium isotopes. The expectation value of $\hat{Q}_{3}$, also known as the octupole moment, is seen in Fig. 4 (top panel) for ${ }^{40} \mathrm{Ca}$ with boost intensity $\varepsilon=1 \times 10^{-8} \mathrm{fm}^{-3}$. When a sine Fourier transformation of this is taken, the octupole strength function (bottom panel in Fig. 4) is produced, resolving the energy spectrum of the $3^{-}$states (see Ref. [16] for details). 

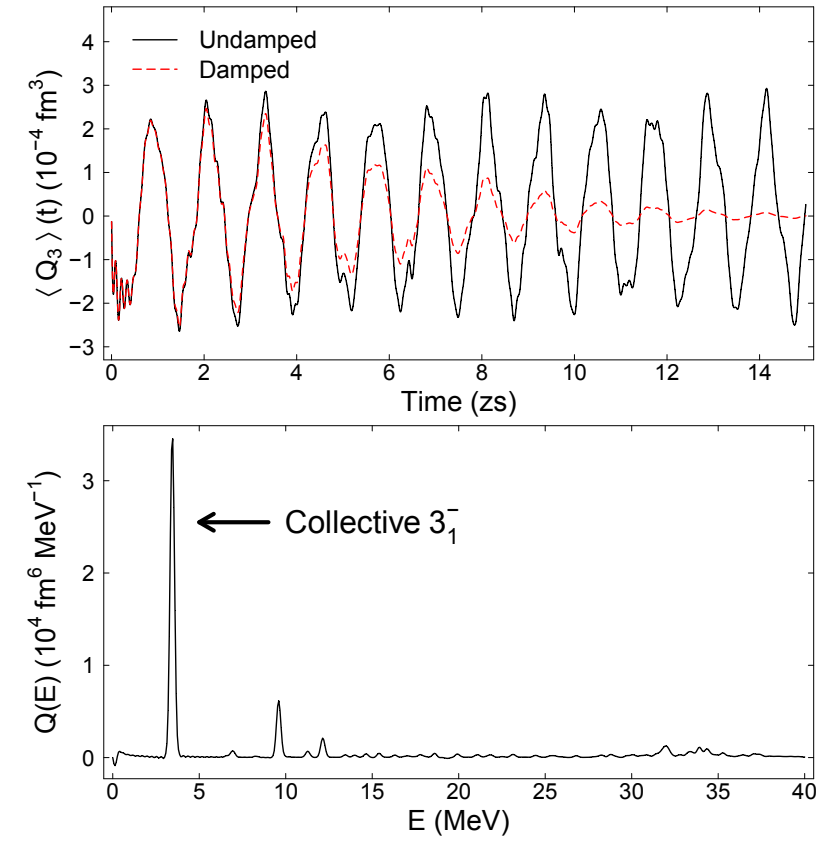

Figure 4. Octupole moment and strength function for ${ }^{40} \mathrm{Ca}$.

The first intense peak of the strength function corresponds to the first collective $3_{1}^{-}$state. The position of the peak is the excitation energy and the area under the peak corresponds to the transition probability, $\left|q_{3}\right|^{2}$, where $q_{3}=\left\langle 3^{-}\left|\hat{Q}_{3}\right| 0\right\rangle$ is the transition amplitude between the ground state $|0\rangle$ and an excited state $\left|3^{-}\right\rangle$. This can be used to determine the deformation parameter according to [66]

$$
\beta_{3}=\frac{4 \pi\left|q_{3}\right|}{3 R_{A}^{3} A},
$$

where $R_{A}$ was taken to be the HF ground state half density radius of the nucleus. In practice, $\left\langle\hat{Q}_{3}\right\rangle(t)$ is multiplied by a damping function to avoid spurious oscillations in the Fourier spectrum due to the finite time of the TDHF calculation.

In Table 1 are the $3-$ state excitation energy and deformation parameter for ${ }^{40} \mathrm{Ca}$, comparing the experimental and TDHF results. Shown in addition to SLy4d and UNEDF1 parametrisations is the SLy4 parametrisation [67] which has the same fitting procedure as for SLy4d but includes the centre of mass correction. This illustrates the large variation in excitation energy amongst different parametrisations. The deformation parameter is slightly underestimated by TDHF in all cases compared to the experimental values. Only the results from the SLy4d parametrisation will be presented from here onwards.

To see what effect just the $3_{1}^{-}$state of the calcium isotopes has on the fusion barriers of the systems, the CC approach [68], using the cCFuLl code [69] is used since dynamical effects can be added in one at a time unlike in TDHF. Couplings to low-lying states could induce a complex structure in the distribution of fusion barriers [2]. A main feature, however, is the lowering of the main peak in the barrier distribution which often results in lowering the average barrier. These average barriers, calculated with

\begin{tabular}{lcc}
\hline \hline & $E_{x}\left(3_{1}^{-}\right)(\mathrm{MeV})$ & $\beta_{3}$ \\
\hline Exp. & 3.737 & $0.3-0.4$ \\
SLy4 & 3.916 & 0.184 \\
SLy4d & 3.442 & 0.224 \\
UNEDF1 & 3.363 & 0.171 \\
\hline \hline
\end{tabular}

Table 1. Comparison of excitation energy and deformation parameter between experimental values [66] and TDHF values for three parametrisations for ${ }^{40} \mathrm{Ca}$.

coupling to the $3_{1}^{-}$state in the calcium isotopes, are shown in by the brown dot-dashed line with stars in Fig. 1. As expected, this coupling lowers the static barrier in all cases. Couplings to low-lying $2^{+}$states (that are not included here) are expected to produce a similar effect.

The inclusion of couplings to low-lying vibrations is helpful for explaining the difference between the static and dynamic cases for the systems with ${ }^{40-50} \mathrm{Ca}$ as projectiles but not for the two most neutron-rich systems. Indeed, we see in Fig. 1 that for the ${ }^{52,54} \mathrm{Ca}+{ }^{116} \mathrm{Sn}$ systems, including dynamics actually increased the fusion barrier as the TDHF fusion threshold is higher than the frozen HF bare potential barrier. This is also the case for ${ }^{54-58} \mathrm{Ca}+{ }^{132} \mathrm{Sn}$ with very little difference (less than $0.5 \mathrm{MeV}$ ) between the two barriers for ${ }^{52-58} \mathrm{Ca}+{ }^{132} \mathrm{Sn}$. Therefore, another dynamical effect aside from vibrations must be considered to help explain this observation.

\subsection{Transfer effects}

Our consideration of transfer is motivated by the fact that the HF dynamic barrier is not consistently lowering the static barrier in the case for the most neutron rich systems. The effect of nucleon transfer acting to balance out the $N / Z$ ratio between two fragments in a reaction is accounted for in TDHF calculations [37, 70].

The $N / Z$ ratio for ${ }^{40-54} \mathrm{Ca}$ ranges from 1 to 1.70 and those of ${ }^{116,132} \mathrm{Sn}$ lie within this range, at 1.32 and 1.64 respectively. For this proceeding, the focus is on proton transfer for these systems. It is expected from the argument of balancing the $N / Z$ ratios that proton transfer would happen in the direction from the light to heavy fragment for the lighter systems, and from heavy to light fragments in neutron rich systems.

Our observation of nucleon transfer in TDHF calculations for the systems ${ }^{40-54} \mathrm{Ca}+{ }^{116,132} \mathrm{Sn}$ occuring at centre of mass energies just below the TDHF fusion threshold $\left(0.999 \% V_{B}\right)$ is from the final fragments. It is quantified by both average numbers of transferred neutrons and protons, and transfer probabilities for protons using the particle number projection method [35]. The average transfer at the end of a reaction, shown by the final $Z$ and $N$ number of the heavy fragment for the systems ${ }^{40-54} \mathrm{Ca}+{ }^{116,132} \mathrm{Sn}$ can be seen in Fig. 5. For a reaction showing near zero values for average transfer (e.g. ${ }^{46} \mathrm{Ca}+{ }^{116} \mathrm{Sn}$ and ${ }^{52} \mathrm{Ca}+{ }^{132} \mathrm{Sn}$ ) there are still possibilities of having non-zero probabilities for other transfer channels.

In the case of ${ }^{116} \mathrm{Sn}$, we can see that the average $Z$ is close to 51 in a reaction with ${ }^{40} \mathrm{Ca}$, corresponding to 


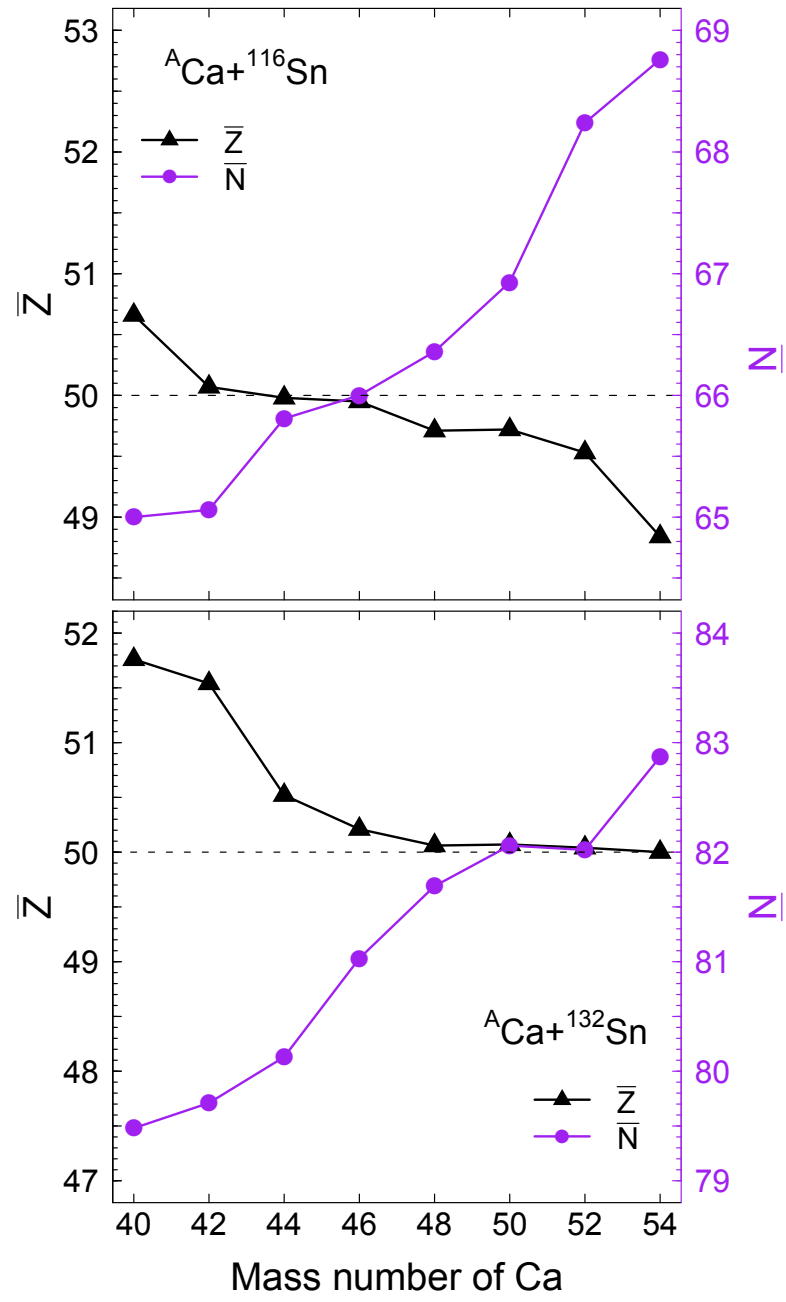

Figure 5. Average $Z$ and $N$ numbers of the heavy fragment at the end of reactions ${ }^{A} \mathrm{Ca}+{ }^{116,132} \mathrm{Sn}$. The original $N$ and $Z$ number of the heavy fragment is indicated by the horizontal dashed line.

proton transfer from calcium to tin. The average $N$ in the same reaction is 65 , indicating one neutron transfer from tin to calcium. The most neutron rich system has transfer of protons and neutrons in the opposite direction and at larger magnitudes.

With ${ }^{132} \mathrm{Sn}$, we see the same direction of proton and neutron transfer as for ${ }^{116} \mathrm{Sn}$ but by different amounts. Very little average proton transfer occurs after ${ }^{48} \mathrm{Ca}+{ }^{132} \mathrm{Sn}$ in agreement with Ref. [34], perhaps because the $N / Z$ ratio of ${ }^{132} \mathrm{Sn}$ is much higher and is a closer match to the heavier calcium nuclei. It was found that subbarier fusion is enhanced for ${ }^{40} \mathrm{Ca}+{ }^{132} \mathrm{Sn}$ more than what can be accounted for by vibrational states [71]. The $Q$-values of nucleon transfer plays an important role in these reactions at sub-barrier energies [71] [ref Back, Liang]. In Ref. [34], the isovector contribution to the DC-TDHF fusion potentials for ${ }^{40,48,54} \mathrm{Ca}+{ }^{132}$ is also related to neutron transfer, compatible with the directions in Fig. 5 and the resulting TDHF fusion barriers in Fig. 3.

In addition to the isovector contribution to the potential, proton transfer in either direction will change the Coulomb repulsion. In particular, one proton transfer from ${ }^{54} \mathrm{Ca}$ to ${ }^{116} \mathrm{Sn}$ increases the Coulomb potential, $V_{C}=$ $e^{2} Z_{1} Z_{2} / r$ by $2.7 \%$ which in turn increases the fusion barrier. This is consistent with what is seen in the TDHF fusion barrier thresholds and is a possible explanation for why the dynamical TDHF fusion threshold is higher than the static frozen HF barrier for this system.

\section{Conclusion}

We have used a microscopic approach to probe fusion reactions involving neutron-rich nuclei. Effects of some static and dynamic structures of the nuclei on fusion were studied using HF methods. In static fusion reactions, properties such as rms neutron radius of the calcium isotopes arising from the HF ground state have an effect on the fusion barrier. Nuclides with less bound neutrons and an increasing neutron skin (calcium isotopes heavier than ${ }^{48} \mathrm{Ca}$ ) lead to a more rapid decrease of the fusion barrier. Including dynamics into reactions, it was seen that for most of the cases this decreased the fusion barrier. However, for the most neutron rich systems, it increased or was very close to the static fusion barrier. The static structure effects disappeared completely with the inclusion of dynamics. Coupled-channels analysis showed that coupling to the $3_{1}^{-}$ state of the calcium nuclei lowered the fusion barrier in all cases. Vibrational states are not enough to describe the fusion barrier differences for the most neutron rich systems, so the significance of other dynamical effects such as proton transfer was discussed. Future works will consider the effect of couplings to low-lying quadrupole vibrations, further understanding the role of neutron transfer on fusion and also incorporating dynamical pairing in the reactions.

\section{Acknowledgement}

This work has been supported by the Australian Research Council under Grant Nos. FT120100760, DP170102423 and FL110100098. The calculations in this proceedings were done with the assistance of resources from the $\mathrm{Na}$ tional Computational Infrastructure (NCI) which is supported by the Australian Government.

\section{References}

[1] N. Rowley et al., Phys. Lett. B 254, 25 (1991)

[2] M. Dasgupta et al., Annu. Rev. Nucl. Part. Sci. 48, 401 (1998)

[3] C.R. Morton et al., Phys. Rev. Lett. 72, 4074 (1994)

[4] J.R. Leigh et al., Phys. Rev. C 52, 3151 (1995)

[5] A.M. Stefanini et al., Phys. Rev. Lett. 74, 864 (1995)

[6] M. Beckerman et al., Phys. Rev. Lett. 45, 1472 (1980)

[7] A. Stefanini et al., Nucl. Phys. A 456, 509 (1986)

[8] H. Timmers et al., Phys. Lett. B 399, 35 (1997)

[9] C.L. Jiang et al., Phys. Rev. C 89, 051603 (2014)

[10] C.L. Jiang et al., Phys. Rev. C 91, 044602 (2015)

[11] M. Dasgupta et al., Phys. Rev. Lett. 82, 1395 (1999)

[12] A. Chatterjee et al., Phys. Rev. Lett. 101, 032701 (2008) 
[13] A. Lemasson et al., Phys. Rev. C 82, 044617 (2010)

[14] A. Lemasson et al., Phys. Lett. B 697, 454 (2011)

[15] J.W. Negele, Rev. Mod. Phys. 54, 913 (1982)

[16] C. Simenel, Eur. Phys. J. A 48, 152 (2012)

[17] T. Nakatsukasa et al., Rev. Mod. Phys. 88, 045004 (2016)

[18] K.H. Kim et al., J. Phys. G: Nucl. Part. Phys. 23, 1267 (1997)

[19] A.S. Umar, V.E. Oberacker, Phys. Rev. C 71, 034314 (2005)

[20] T. Nakatsukasa, K. Yabana, Phys. Rev. C 71, 024301 (2005)

[21] J.A. Maruhn et al., Phys. Rev. C 71, 064328 (2005)

[22] C. Simenel, P. Chomaz, Phys. Rev. C 80, 064309 (2009)

[23] I. Stetcu, A. Bulgac et al., Phys. Rev. C 84, 051309 (2011)

[24] S. Fracasso et al., Phys. Rev. C 86, 044303 (2012)

[25] B. Avez, C. Simenel, Eur. Phys. J. A 49, 76 (2013)

[26] G. Scamps, D. Lacroix, Phys. Rev. C 88, 044310 (2013)

[27] C. Simenel et al., Phys. Rev. C 88, 024617 (2013)

[28] C. Simenel et al., Phys. Rev. C 88, 064604 (2013)

[29] A.S. Umar et al., Phys. Rev. C 89, 034611 (2014)

[30] G.F. Dai et al., Phys. Rev. C 90, 044609 (2014)

[31] K. Washiyama, Phys. Rev. C 91, 064607 (2015)

[32] D. Bourgin et al., Phys. Rev. C 93, 034604 (2016)

[33] K. Vo-Phuoc et al., Phys. Rev. C 94, 024612 (2016)

[34] K. Godbey et al., Phys. Rev. C 95, 011601 (2017)

[35] C. Simenel, Phys. Rev. Lett. 105, 192701 (2010)

[36] C. Simenel, Phys. Rev. Lett. 106, 112502 (2011)

[37] K. Sekizawa, K. Yabana, Phys. Rev. C 88, 014614 (2013)

[38] K. Sekizawa, K. Yabana, Phys. Rev. C 90, 064614 (2014)

[39] G. Scamps, D. Lacroix, Phys. Rev. C 87, 014605 (2013)

[40] A. Wakhle et al., Phys. Rev. Lett. 113, 182502 (2014)

[41] V.E. Oberacker et al., Phys. Rev. C 90, 054605 (2014)

[42] K. Hammerton et al., Phys. Rev. C 91, 041602 (2015)
[43] A.S. Umar et al., Phys. Rev. C 92, 024621 (2015)

[44] E. Prasad et al., Phys. Rev. C 93, 024607 (2016)

[45] K. Sekizawa, K. Yabana, Phys. Rev. C 93, 054616 (2016)

[46] A.S. Umar et al., Phys. Rev. C 94, 024605 (2016)

[47] C. Simenel, A.S. Umar, Phys. Rev. C 89, 031601 (2014)

[48] G. Scamps et al., Phys. Rev. C 92, 011602 (2015)

[49] P. Goddard et al., Phys. Rev. C 92, 054610 (2015)

[50] P. Goddard et al., Phys. Rev. C 93, 014620 (2016)

[51] A. Bulgac et al., Phys. Rev. Lett. 116, 122504 (2016)

[52] Y. Tanimura et al., Phys. Rev. Lett. 118, 152501 (2017)

[53] C. Simenel et al., Phys. Rev. C 95, 031601 (2017)

[54] M. Kortelainen et al., Phys. Rev. C 89, 054314 (2014)

[55] M. Kortelainen et al., Phys. Rev. C 85, 024304 (2012)

[56] P. Bonche et al., Comp. Phys. Com. 171, 49 (2005)

[57] S.J. Krieger et al., Nucl. Phys. A 517, 275 (1990)

[58] M. Bender et al., Phys. Rev. C 60, 034304 (1999)

[59] K. Washiyama, D. Lacroix, Phys. Rev. C 78, 024610 (2008)

[60] C. Simenel, B. Avez, International Journal of Modern Physics E 17, 31 (2008)

[61] R.A. Broglia, A. Winther, Heavy Ion Reaction Lecture Notes, Vol. I: Elastic and Inelastic Reactions (Benjamin/Cummings, Reading, MA, 1981)

[62] H.D. Wohlfahrt et al., Phys. Rev. C 23, 533 (1981)

[63] L. Vermeeren et al., Phys. Rev. Lett. 68, 1679 (1992)

[64] E. Caurier et al., Phys. Lett. B 522, 240 (2001)

[65] K. Hagino, N. Takigawa, Phys. Rev. Lett. 79, 2014 (1997)

[66] T. Kibedi, R.H. Spear, Atomic Nuclear and Data Tables 80, 35 (2002)

[67] E. Chabanat et al., Nucl. Phys. A 635, 231 (1998)

[68] K. Hagino, N. Takigawa, Prog. Th. Phys. 128, 1061 (2012)

[69] K. Hagino et al., Comp. Phys. Com. 123, 143 (1999)

[70] C. Simenel et al., Phys. Lett. B 710, 607 (2012)

[71] J.J. Kolata et al., Phys. Rev. C 85, 054603 (2012) 